\title{
Parametric Macromodeling for Tabulated Data Based on Internal Passivity
}

\author{
Francesco Ferranti, Member, IEEE, Luc Knockaert, Senior Member, IEEE, Tom Dhaene, Senior Member, IEEE, \\ Giulio Antonini, Senior Member, IEEE and Daniel De Zutter, Fellow, IEEE
}

\begin{abstract}
We present a new parametric macromodeling technique for admittance and impedance input-output representations parameterized by design variables such as geometric and material parameters. Stability and passivity of the parametric macromodel are guaranteed over a user defined range of design parameter values, while poles and residues are parameterized indirectly. Numerical results validate the proposed parametric macromodeling approach.
\end{abstract}

Index Terms-Parametric macromodeling, rational approximation, interpolation, passivity, positive real lemma.

\section{INTRODUCTION}

Robust parametric macromodeling is becoming increasingly important for efficient design space exploration, design optimization and sensitivity analysis of electromagnetic (EM) systems. These design activities require multiple simulations for different design parameter values. It is often not feasible to perform multiple simulations of large circuits due to variations of these parameters. Such design activities call for the development of robust parametric macromodeling techniques. Parametric macromodels can take multiple design variables into account, such as geometrical layout or substrate features and are obtained through an identification process starting from a set of multivariate data samples.

The development of parametric macromodels of EM systems has become a topic of intense research over the last years. Some parametric macromodeling techniques parameterize both poles and residues [1], [2] and are able to accurately model highly dynamic multivariate data samples without requiring a high sample density. Unfortunately, such techniques are not able to guarantee overall stability and passivity of the parametric macromodel. In other formulations, poles are kept fixed and only residues are parameterized [3], [4]; the modeling power is reduced with respect to the previous techniques, but the preservation of stability and passivity of the parametric macromodel is feasible.

This paper presents a novel parametric macromodeling technique that parameterizes poles and residues to own a high modeling power and is able to guarantee stability and passivity over the entire design space, for admittance $(\mathbf{Y})$ and

Manuscript received March 4, 2010; revised July 9, 2010.

Francesco Ferranti, Luc Knockaert, Tom Dhaene and Daniel De Zutter are with the Department of Information Technology (INTEC), at Ghent University - IBBT, Sint Pietersnieuwstraat 41, 9000 Ghent, Belgium, email: \{francesco.ferranti, luc.knockaert, tom.dhaene, daniel.dezutter\}@ugent.be.

Giulio Antonini is with the UAq EMC Laboratory, Dipartimento di Ingegneria Elettrica e dell'Informazione, Università degli Studi dell'Aquila, Via G. Gronchi 18, 67100, L'Aquila, Italy, e-mail: giulio.antonini@univaq.it.

This work was supported by the Research Foundation Flanders (FWO). impedance $(\mathbf{Z})$ representations. It turns out that the choice of a direct parameterization of poles and residues is not appropriate, due to their possible highly non-smooth behavior with respect to the design parameters. The direct parameterization of poles and residues is avoided in our new technique, where some state-space matrices are parameterized and, hence, also poles and residues indirectly, while stability and passivity are guaranteed over the design space of interest. The proposed technique is validated by a pertinent numerical example.

\section{PARAMETRIC MACROMOdEling}

This section treats the generation of a multivariate representation $\mathbf{R}(s, \vec{g})$ which accurately models a large set of $K_{\text {tot }}$ multivariate data samples $\left\{(s, \vec{g})_{k}, \mathbf{H}(s, \vec{g})_{k}\right\}_{k=1}^{K_{\text {tot }}}$, while preserving stability and passivity over the entire design space. These data samples depend on the complex frequency $s=j \omega$, and several design variables $\vec{g}=\left(g^{(m)}\right)_{m=1}^{M}$, such as layout features or substrate parameters. Since a rational formulation (poles/residues, zeros/poles, or numerator/denominator polynomials) suffers from severe ill-conditioning when the dependence of external parameters is included, a parametric macromodel in the form

$$
\mathbf{R}(s, \vec{g})=\frac{\sum_{n=0}^{N} \mathbf{R}_{n}(\vec{g}) \phi_{n}(s)}{\sum_{n=0}^{N} r_{n}(\vec{g}) \phi_{n}(s)}
$$

is adopted [2]. The basis function $\phi_{n}(s)$ with the predefined maximal order $N$ are used to describe the frequency dependence of the system under study, while parameters variations are induced by their expansion coefficients $\mathbf{R}_{n}(\vec{g}), r_{n}(\vec{g})$. The frequency-dependent basis functions $\phi_{n}(s)$ are chosen in the partial fraction form [5]

$$
\begin{aligned}
& \phi_{0}(s)=1, n=0 \\
& \phi_{n}(s)=\frac{1}{s-a_{n}}, n=1, \ldots, N
\end{aligned}
$$

since these basis functions are numerically reliable.

\section{A. Root Macromodels}

Starting from a set of data samples $\left\{(s, \vec{g})_{k}, \mathbf{H}(s, \vec{g})_{k}\right\}_{k=1}^{K_{t o t}}$ a frequency-dependent rational model is built for some grid points in the design space by means of the Vector Fitting (VF) technique [5]. A pole-flipping scheme is used to enforce stability [5], while passivity enforcement can be accomplished using one of the robust standard techniques [6], [7]. The result of this 
initial procedure is a set of rational univariate macromodels, stable and passive, that we call root macromodels. These are the starting points to build a parametric macromodel. Each root macromodel related to a generic point $\vec{g}_{k}=\left(g_{k_{1}}^{(1)}, \ldots, g_{k_{M}}^{(\mathrm{M})}\right)$ in the design space is converted from the rational pole-residue form

$$
\mathbf{R}_{\vec{g}_{k}}(s)=\mathbf{C}_{0, \vec{g}_{k}}+\sum_{n=1}^{N} \frac{\mathbf{C}_{n, \vec{g}_{k}}}{s-p_{n, \vec{g}_{k}}}
$$

obtained by means of VF, into the barycentric form [2], [8]

$$
\mathbf{R}_{\vec{g}_{k}}(s)=\frac{\mathbf{F}_{0, \vec{g}_{k}}+\sum_{n=1}^{N} \frac{\mathbf{F}_{n, \vec{g}_{k}}}{s-a_{n}}}{f_{0, \vec{g}_{k}}+\sum_{n=1}^{N} \frac{f_{n, \vec{g}_{k}}}{s-a_{n}}}
$$

The basis poles $\left\{a_{n}\right\}_{n=1}^{N}$ affect the numerical conditioning of the basis functions $\phi_{n}(s)$ and the conversion from the VF form (3) to the barycentric form (4). The proposed set of rational bases (2a)-(2b) is well conditioned if the basis poles are linearly distributed over the available bandwidth [5]. Consequently, we adopt this rule to define the basis poles $\left\{a_{n}\right\}_{n=1}^{N}$. A state-space realization is performed for each root macromodel

$$
\mathbf{R}_{\vec{g}_{k}}(s)=\mathbf{C}_{\vec{g}_{k}}\left(s \mathbf{I}-\mathbf{A}_{\vec{g}_{k}}\right)^{-1} \mathbf{B}_{\vec{g}_{k}}+\mathbf{D}_{\vec{g}_{k}}
$$

after the conversion from the VF form (3) to the barycentric form (4).

\section{B. Internally Passive Realization}

When performing transient analysis, stability and passivity must be guaranteed. It is known that, while a passive system is also stable, the reverse is not necessarily true [9], which is crucial when the macromodel is to be utilized in a generalpurpose nonlinear simulator. Once a state-space realization for each stable and passive root macromodel is computed, a conversion from (5) to an internally passive realization [10] is performed using the solution $\mathbf{P}=\mathbf{P}^{\mathrm{T}}>0$ of the linear matrix inequality (LMI) [11] associated to the positive real lemma [12], [13]

$$
\left[\begin{array}{cc}
\mathbf{A}^{\mathrm{T}} \mathbf{P}+\mathbf{P A} & \mathbf{P B}-\mathbf{C}^{\mathrm{T}} \\
\mathbf{B}^{\mathrm{T}} \mathbf{P}-\mathbf{C} & -\mathbf{D}-\mathbf{D}^{\mathrm{T}}
\end{array}\right] \leq 0, \mathbf{P}=\mathbf{P}^{\mathrm{T}}>0
$$

which is a convex formulation. The LMI (6) can be solved via convex optimization [11]. Once a $\mathbf{P}$ matrix is computed for each root macromodel, it is factorized into $\mathbf{S}^{\mathrm{T}} \mathbf{S}=\mathbf{P}$ with $\mathbf{S}=\sqrt{\mathbf{P}}$ nonsingular and the state-space conversion from $\left\{\mathbf{A}_{\vec{g}_{k}}, \mathbf{B}_{\vec{g}_{k}}, \mathbf{C}_{\vec{g}_{k}}, \mathbf{D}_{\vec{g}_{k}}\right\}$ to $\left\{\widetilde{\mathbf{A}}_{\vec{g}_{k}}, \widetilde{\mathbf{B}}_{\vec{g}_{k}}, \widetilde{\mathbf{C}}_{\vec{g}_{k}}, \widetilde{\mathbf{D}}_{\vec{g}_{k}}\right\}=$ $\left\{\mathbf{S A}_{\vec{g}_{k}} \mathbf{S}^{-1}, \mathbf{S B}_{\vec{g}_{k}}, \mathbf{C}_{\vec{g}_{k}} \mathbf{S}^{-1}, \mathbf{D}_{\vec{g}_{k}}\right\}$ is performed. The internally passive realization $\left\{\widetilde{\mathbf{A}}_{\vec{g}_{k}}, \widetilde{\mathbf{B}}_{\vec{g}_{k}}, \widetilde{\mathbf{C}}_{\vec{g}_{k}}, \widetilde{\mathbf{D}}_{\vec{g}_{k}}\right\}$ has the property [10]

$$
\begin{gathered}
\mathbf{G}_{\vec{g}_{k}}=\left[\begin{array}{cc}
-\widetilde{\mathbf{A}}_{\vec{g}_{k}} & -\widetilde{\mathbf{B}}_{\vec{g}_{k}} \\
\widetilde{\mathbf{C}}_{\vec{g}_{k}} & \widetilde{\mathbf{D}}_{\vec{g}_{k}}
\end{array}\right] \\
\mathbf{G}_{\vec{g}_{k}}+\mathbf{G}_{\vec{g}_{k}}^{\mathrm{T}} \geq 0
\end{gathered}
$$

\section{Passivity Preserving Interpolation}

The next step of the parametric macromodeling algorithm is focused on gluing together the computed internally passive realizations of the root macromodels by a multivariate interpolation scheme that can guarantee stability and passivity over the entire design space by preserving the positive semidefiniteness of the matrix $\mathbf{G}(\vec{g})+\mathbf{G}(\vec{g})^{\mathrm{T}}$.

Any square matrix $\mathbf{A}$ can be written as the sum of a symmetric and a skew-symmetric matrix, $\mathbf{A}=\mathbf{A}_{1}+\mathbf{A}_{2}$, where $\mathbf{A}_{1}=\left(\mathbf{A}+\mathbf{A}^{\mathrm{T}}\right) / 2$ and $\mathbf{A}_{2}=\left(\mathbf{A}-\mathbf{A}^{\mathrm{T}}\right) / 2$. We recall that the square root and square matrix operators applied to any positive semidefinite matrix provide a positive semidefinite matrix. The symmetric part of $\mathbf{G}(\vec{g})$ has to be positive semidefinite, therefore the square root matrix operator is applied to the set of matrices $\left(\mathbf{G}_{\vec{g}_{k}}+\mathbf{G}_{\vec{g}_{k}}^{\mathrm{T}}\right) / 2$, and only the lower or upper triangular part is interpolated. Any multivariate interpolation scheme can be used, e.g. the piecewise multilinear and multivariate simplicial methods [14], the multivariate cubic splines method [15]. Finally, the interpolated matrix is mapped back by the square matrix operator resulting in a positive semidefinite matrix $\mathbf{G}(\vec{g})+\mathbf{G}(\vec{g})^{\mathrm{T}}$. Regarding the skew-symmetric parts $\left(\mathbf{G}_{\vec{g}_{k}}\right.$ $\mathbf{G}_{\vec{g}_{k}}^{\mathrm{T}}$ )/2, only the strictly lower (or strictly upper) triangular parts are directly interpolated by any interpolation scheme. Once the matrix $\mathbf{G}(\vec{g})$ is modeled, it is straightforward to obtain $\{\widetilde{\mathbf{A}}(\vec{g}), \widetilde{\mathbf{B}}(\vec{g}), \widetilde{\mathbf{C}}(\vec{g}), \widetilde{\mathbf{D}}(\vec{g})\}$.

\section{NUMERICAL EXAMPLE}

\section{One Microstrip With Variable Length}

In this example a microstrip has been modeled. The conductor has width $W=100 \mu \mathrm{m}$ and the substrate has height $h=300 \mu \mathrm{m}$. A bivariate macromodel is built as a function of the length $L$ of the line in addition to frequency. Their corresponding ranges are freq $=\left[1 \cdot 10^{-2}-10\right] \mathrm{GHz}$ and $L=[17-22] \mathrm{mm}$.

The admittance matrix $\mathbf{Y}(s, L)$ has been computed over a validation grid of $200 \times 21$ samples $($ freq, $L)$ by means of a full-wave solver. We have built root macromodels for 8 values of the length by means of VF, each with a order equal to 8 . The passivity of each model has been verified by checking the eigenvalues of the Hamiltonian matrix [11] and enforced, if needed. Both enforcing passivity on the root macromodels and using the passivity preserving interpolation method described in Section II-C ensure the passivity of the parametric macromodel over the entire design space. If one of these two conditions is not satisfied, the overall passivity of the parametric macromodel cannot be guaranteed. Then, the numerical manipulations described in Section II are used to obtain an internally passive realization for each root macromodel. Finally, a bivariate macromodel is obtained by spline interpolation. Fig. 1 shows the magnitude of the parametric macromodel of $\mathbf{Y}_{12}(s, L)$. The worst case weighted RMSerror over the validation grid [3] is equal to $2.1 \cdot 10^{-2}$ and it occurs for $g_{\max }=L=21.3 \mathrm{~mm}$. Fig. 2 shows the minimum eigenvalue of (8) as a function of $L$ and confirms the overall passivity, and hence stability, of the parametric macromodel. Fig. 3 shows the distribution of the poles of the parametric macromodel in the $s$-plane as a function of the 
length $L$. As clearly seen, a very good agreement is obtained between the original data and the proposed passivity preserving macromodeling technique. The parametric macromodel captures the behavior of the system very accurately, while preserving stability and passivity over the entire design space.

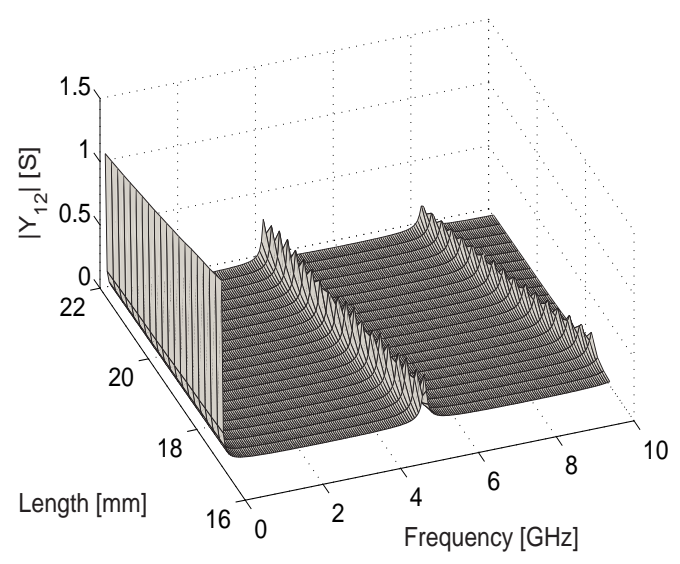

Fig. 1. Magnitude of the parametric macromodel of $\mathbf{Y}_{12}(s, L)$.

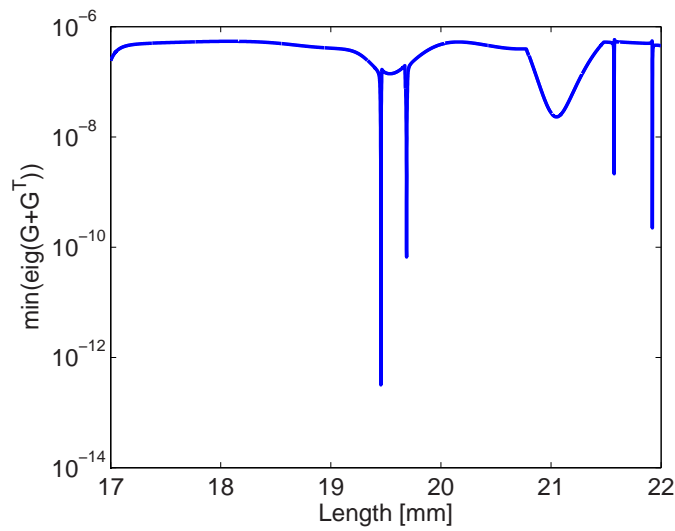

Fig. 2. Minimum eigenvalue of (8) as a function of $L$ (10.000 $L$ points).

\section{CONCLUSiOnS}

We have proposed a novel parametric macromodeling technique for the generation of parametric macromodels of admittance and impedance representations. The indirect parameterization of poles and residues provides a high power modeling, while overall stability and passivity are guaranteed by applying a special kind of interpolation on some state-space matrices related to internally passive realizations. A numerical example illustrates the capability of the algorithm to model highly dynamic parameterized frequency responses very accurately, while guaranteeing stability and passivity over the complete design space.

\section{REFERENCES}

[1] D. Deschrijver, T. Dhaene and D. De Zutter, "Robust parametric macromodeling using multivariate orthonormal vector fitting," IEEE Trans. Microw. Theory Tech., vol. 56, no. 7, pp. 1661-1667, July 2008.
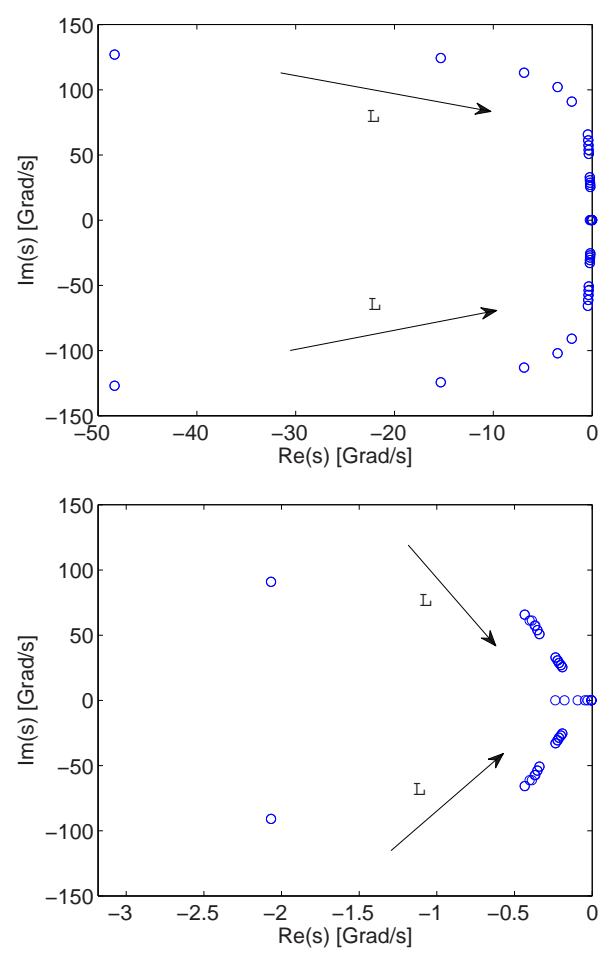

Fig. 3. Model poles as a function of $L$, overall view (top), zoom view (bottom).

[2] P. Triverio, S. Grivet-Talocia, and M. S. Nakhla, "A parameterized macromodeling strategy with uniform stability test," IEEE Trans. Adv. Packag., vol. 32, no. 1, pp. 205-215, Feb. 2009.

[3] F. Ferranti, L. Knockaert, and T. Dhaene, "Guaranteed passive parameterized admittance-based macromodeling," IEEE Trans. Adv. Packag., to be published.

[4] _ - "Parameterized S-parameter based macromodeling with guaranteed passivity," IEEE Microw. Wireless Compon. Lett., vol. 19, no. 10, pp. 608-610, Oct. 2009.

[5] B. Gustavsen and A. Semlyen, "Rational approximation of frequency domain responses by vector fitting," IEEE Trans. Power Delivery, vol. 14, no. 3, pp. 1052-1061, July 1999.

[6] D. Saraswat, R. Achar and M. S. Nakhla, "Global passivity enforcement algorithm for macromodels of interconnect subnetworks characterized by tabulated data," IEEE Transactions on Very Large Scale Integration (VLSI) Systems, vol. 13, no. 7, pp. 819 - 832, July 2005.

[7] B. Gustavsen, "Fast passivity enforcement for pole-residue models by perturbation of residue matrix eigenvalues," IEEE Trans. Power Delivery, vol. 23, no. 4, pp. 2278-2285, Oct. 2008.

[8] L. Knockaert, F. Ferranti, and T. Dhaene, "Vector fitting vs. levenbergmarquardt : Some experiments," in Proc. IEEE Workshop on Signal Propagation on Interconnects SPI '09, May 12-15, 2009, pp. 1-4.

[9] R. Rohrer, H. Nosrati, "Passivity considerations in stability studies of numerical integration algorithms," IEEE Transactions on Circuits and Systems, no. 9, pp. 857-866, Sept. 1981.

[10] J. Willems, "Realization of systems with internal passivity and symmetry constraints," J. Franklin Inst., vol. 301, no. 6, pp. 605-621, 1976.

[11] S. Boyd, L. El Ghaoui, E. Feron and V. Balakrishnan, Linear Matrix Inequalities in System and Control Theory. Philadelphia, PA: SIAM, 1994, vol. 15

[12] B. D. Anderson, S. Vongpanitlerd, Network Analysis and Synthesis. Englewood Cliffs, NJ: Prentice-Hall, 1973.

[13] R. F. Curtain, "Old and new perspectives on the positive-real lemma in systems and control theory," ZAMM, vol. 79, no. 9, pp. 579-590, 1999.

[14] W. A. Weiser and S. E. Zarantonello, "A note on piecewise linear and multilinear table interpolation in many dimensions," Mathematics of Computation, vol. 50, no. 181, pp. 253-264, Jan. 1988.

[15] C. de Boor, A practical Guide to Splines. Springer-Verlag, 2001. 